\title{
DROPLET-BETWEEN-ELECTRODES FOR ULTRAHIGH INTERFACIAL CAPACITIVE SENSING
}

\author{
B. Nie ${ }^{1}$, S. Xing ${ }^{1}$, J.D. Brandt ${ }^{2}$, and T. Pan ${ }^{1 *}$ \\ ${ }^{1}$ Department of Biomedical Engineering, University of California, Davis, USA \\ ${ }^{2}$ Department of Ophthalmology, University of California, Davis, Health System, USA
}

\begin{abstract}
In this paper we have presented a novel droplet-based interfacial capacitive sensor, achieving ultrahigh mechanical-to-electrical sensitivity $(1.58 \mathrm{~F} / \mathrm{kPa})$ and resolution (1.8Pa) using a simple device architecture. The miniature transparent droplet sensors consisted of two flexible polymer membranes with conductive coating and a separation layer hosting a sensing chamber for an electrolyte droplet. The sensing principle primarily relied on high elasticity of the sensing droplet and large capacitance presented at the electrode-electrolyte interface. Theoretical analyses and experimental investigations on several design parameters were thoroughly conducted to characterize and optimize the overall sensitivity of the device. Finally, the droplet sensor was successfully applied to detect minute blood pressure variations on the skin surface throughout cardiovascular cycles.
\end{abstract}

\section{INTRODUCTION}

Capacitive sensing has received increasing attention from academia, industry, and biomedical application due to its high electrical sensitivity, low power consumption, simple device construction and immunity to temperature fluctuation in comparison with its resistive counterparts. For example, Bao's group recently reported an elastomer-based capacitive sensor to achieve high sensitivity and flexibility [1], while Ezzat, et al., sandwiched a mercury droplet between two electrodes covered by an ultrahigh-permittivity material to detect the change of the contact area under external loads [2]. Moreover, Wise's group devised a capacitive pressure sensor in a wireless configuration to monitor intraocular pressure [3]. The capacitive sensing module, using conventional silicon-based microfabrication integrated with processing circuitry, was fully encapsulated in a transparent glass diaphragm which allowed continuous recording of intraocular pressure once implanted.

Recently, the electric double layer (EDL) between conductive liquid and solid phases has been shown to yield ultrahigh capacitance per unit area, typically on the order of $10 \mathrm{~F} / \mathrm{cm}^{2}$ [4]. The unique EDL interface has been explored for energy storage, interfacial manipulation, and biomolecule sensing. For example, the EDL has been used as an energy hoarder in the field of electrical energy storage and harvesting applications. In the contacts of nanostructured carbon electrodes and ionic liquids, EDL capacitance are able to operate in a wide of temperature range (from $-50^{\circ} \mathrm{C}$ to $100^{\circ} \mathrm{C}$ ) at very high charge/discharge rates (of up to $20 \mathrm{~V} / \mathrm{s}$ ), solving the significant limitation for the batteries relying on redox reactions [5]. In addition, EDL capacitance has been introduced for the detection of human serum albumin (HSA) concentration, according to the changes of the electrical impedances on the anti-HSA-coated gold electrode surfaces [6].

This paper reports a novel droplet-based capacitive sensing mechanism utilizing the highly mechanically deformable elastic and electrically conductive droplet-solid interfaces with ultrahigh unit-area capacitance from the EDL capacitance at the solid-liquid interface. The miniature transparent capacitive pressure sensor achieves an ultrahigh pressure sensitivity of $1.58 \mathrm{~F} / \mathrm{kPa}$ along with ultrahigh resolution of $1.8 \mathrm{~Pa}$, illustrated in Fig. 1, in which the sensing droplets are dyed with various colors for improved visibility.

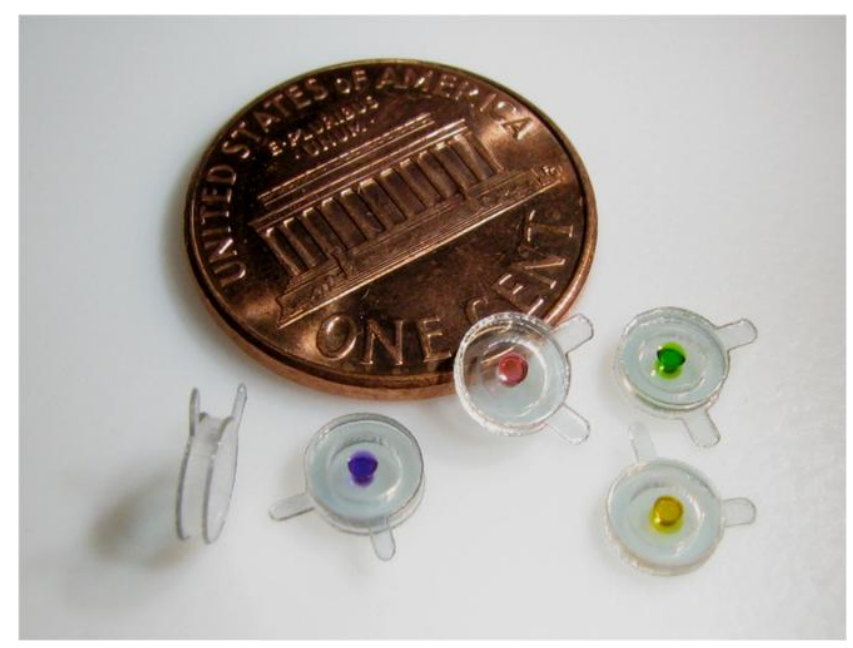

Figure 1: Ultrahigh interfacial capacitive sensors (dyed with different colors).

\section{THEORY}

The interfacial capacitive sensor consists of two flexible polymer membranes with conductive coating and a separation layer hosting an electrolyte droplet, forming a sensing chamber, as illustrated in Fig. 2a. Fig. 2b shows the equivalent circuit diagram, where the interfacial EDL capacitances at the electrolyte-electrode interface are modeled as two capacitors connected in series through the resistive element of the conductive droplet. Under external loads, the suspended membrane and the separation layer deform elastically, and as a result, the interfacial contact area experiences circumferential expansion. Given a relatively constant unit-area capacitance $\left(c_{0}\right)$ of the interfacial EDL, the variation in the contact area will lead to a proportional change in the EDL capacitance $\left(C_{E D L}\right)$ so that the sensor produces a linear capacitive response to pressure changes. The mechanical-to-electrical sensitivity of the sensing device can be analytically expressed as:

$$
\frac{\Delta \mathrm{C}_{\mathrm{EDL}}}{\mathrm{P}}=\mathrm{c}_{\mathrm{o}}\left(\alpha \mathrm{R}^{2}+\beta \mathrm{H}\right) \frac{\mathrm{V}_{\mathrm{d}} \mathrm{R}^{2}}{\mathrm{H}^{2}}
$$

where $P$ is mechanical load. $\alpha$ and $\beta$ represent the membrane deflection and the elastic deformation of the separation layer, and can be determined by the geometrical and mechanical properties of the sensing membrane and the separation layer, respectively. $R$ and $H$ represent the radius and height of the sensing chamber, while $V_{d}$ indicates the volume of the electrolyte droplet. As can be seen, both the membrane deflection (the 1st term) and the separation layer deformation (the 2nd term) contribute to the overall mechanical-to-capacitive sensitivity. It is also worth noting that both the hydraulic pressure of the droplet and the radius of the curvature at the droplet-electrode interface have been ignored in the simplified mathematical expression. In addition, the gravitational effect has been neglected, since the droplet volume is typically confined to the ones smaller than the cube of the capillary length. 


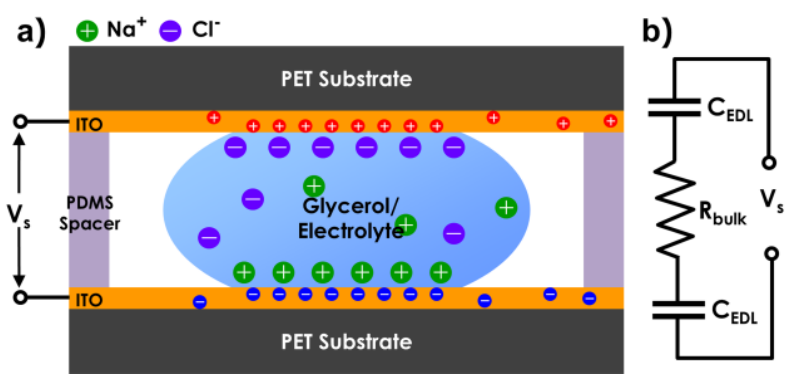

Fig. 2 a) Illustration of the droplet-based interfacial capacitive sensing principle, and b) the equivalent electrical circuit diagram.

\section{METHODS}

\section{Surface Modification}

Indium-tin-oxide (ITO)-coated polyethylene terephthalate (PET) films (Southwall Technology) were used to serve as the pressure sensing membranes. In order to reduce the hysteresis of the droplet deformation, simple surface modification was introduced to the conductive coating. In brief, the ITO-coated PET sheets were exposed to oxygen plasma at $30 \mathrm{~W}$ for $30 \mathrm{sec}$ for surface hydroxylation. Following the plasma activation, a dielectric polydimethylsiloxane (PDMS) stamp was brought into physical contact with the conductive coating enriched with hydroxyl groups for 2 hours, during which a nanometer-thick layer of PDMS oligomers was transferred and immobilized onto the electrode surface [7]. In addition, an indented hole (of $200 \mathrm{~m}$ in diameter) was included in the center of the PDMS stamp, leaving a hydrophilic spot surrounded by the hydrophobic oligomer layer on the electrode surface, by which the sensing droplet could be anchored and stabilized in the middle of the sensing chamber.

\section{Device Fabrication}

To fabricate the device components, ITO-coated PET films and a PDMS layer were laser-micromachined to serve as the sensing membranes and the separation layer, respectively. In the subsequent step, the laser-trimmed PET substrates with ITO coating enriched with PDMS oligomers as aforementioned were bonded to the PDMS separation layer through an oxygen plasma-assisted bonding. Prior to the final assembly of the device, an electrolyte droplet with a desired volume was dispensed by a micropipette in the centre of the sensing chamber.

\section{Electrical and Mechanical Characterization}

External mechanical point loads were applied onto the centre of the sensing membrane through a custom-built motorized force gauge with $1 \mathrm{mN}$ resolution (DFS, Chatillon), driven by a computer-controlled step motor (VT-80, Micos) with a spatial resolution of $0.2 \mathrm{~m}$. The mechanical-to-capacitance responses, measured by LCR meter, were evaluated twice on two identical devices for each parameter. For the resolution measurement, minute droplets (of 50 L volume) dispensed by a micropipette was applied directly onto the sensing membrane till a noticeable capacitive change appeared in the LCR meter, which was evaluated three times for the same device.

\section{RESULTS}

\section{Sensing Droplets}

As a vital part of the interfacial sensing device, physical properties of the sensing droplet have to satisfy following criteria: high ionic concentration (ensuring high electrical conductance and interfacial capacitance), polarized molecular structure (reversible elasticity on the hydrophobic surfaces) and low fluidic viscosity (allowing rapid mechanical response). Aqueous-based electrolyte solution (e.g., $\mathrm{NaCl}$ ) with high ionic concentration can be a natural choice, satisfying all except for moderate evaporation under a room condition. According to a previous study, mixing an aqueous solution with glycerol can effectively reduce evaporation due to decreased vapor pressure [8]. Through examining the physical properties (conductivity, evaporation, and viscosity) of the electrolyte/glycerol $(\mathrm{E} / \mathrm{G})$ mixture (given a $\mathrm{NaCl}$ solution at the concentration of $1.1 \mathrm{~mol} / \mathrm{L})$ at various mixing ratios $(\mathrm{v} / \mathrm{v} \%)$, an optimal mixing ratio of $25 / 75 \% \mathrm{E} / \mathrm{G}$ solution has been chosen as the working fluid for the sensing droplet, considering its highly conductive and non-evaporative under a laboratory condition $(46 \%$ humidity and $24^{\circ} \mathrm{C}$ ).

\section{Surface Modification}

As aforementioned, surface hydrophobic treatment has been applied to the conductive coating, which ensures the reversible and elastic deformability of the sensing interface. The surface energy during the oligomer transfer process is compared by measuring the contact angle of a mixed E/G solution (25/75\%). With an oxygen-plasma treatment, the ITO coating is rendered apparent hydrophilic (the contact angle reduces from $66^{\circ}$ to $15^{\circ}$ ). Following the oligomer transfer step, the PDMS oligmer-coated ITO surface switches the polarity from hydrophilicity to hydrophobicity with the contact angle of $90^{\circ}$, which further enhances the elasticity and reversibility of the droplet-electrode contact by reducing adhesive energy of the liquid to the substrate.

\section{Mechanical-to-Capacitive Sensitivity}

The overall mechanical-to-electrical sensitivity $\left(\Delta C_{E D L} / P\right)$ can be determined by the geometrical confinements (the radius $R$ and height $H$ of the sensing chamber and the thickness of the polymeric membrane), droplet volume $\left(V_{d}\right)$, and the material properties of the construct (Young's modulus and Poisson's ratio), given a fixed unit-area capacitance $\left(c_{o}\right)$. Among those, the radius of the sensing chamber play the most important role, followed by the height of the chamber, as predicted in the Theory. In addition, the system sensitivity is directly proportional to the volume of the sensing droplet. Experimental investigations have been conducted to verify the above theoretical predications.
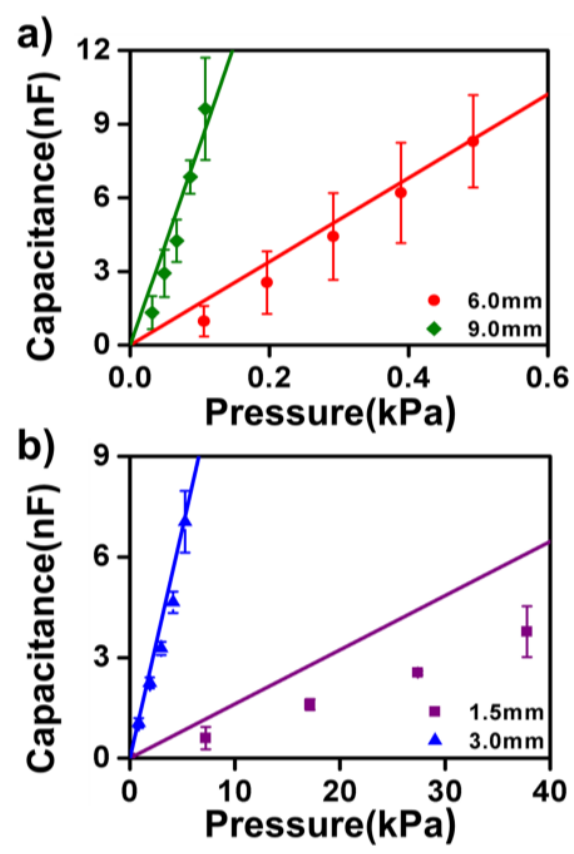

Fig. 3 Influence of different radii of the sensing chambers on the device sensitivity. 


\section{Influence of Chamber Radius}

Fig. 3a and Fig. 3b illustrate the capacitive changes over a wide spectrum of pressure loaded on the droplet sensors with different sizes of the sensing chamber, of which the radius varies from 1.5 to $9.0 \mathrm{~mm}$, given the chamber height of $200 \mathrm{~m}$ and the droplet volume of $0.3 \mathrm{~L}$. The experimental measurements (dots) are plotted in comparison with the values calculated from Eq. 1 (curves), and the slope rate of each device measurement defines the corresponding device sensitivity. As predicted by the sensing theory, the radius exhibits 4th power in membrane deflection and 2 nd power in the elastic deformation. Within the small deflection limit, the capacitive charges change linearly with the external load as one would expect. In the devices with the largest sensing chamber (of $9.0 \mathrm{~mm}$ radius), the highest sensitivity of $90.2 \mathrm{nF} / \mathrm{kPa}$ has been achieved. As the radius is reduced to two thirds of the former one $(6.0 \mathrm{~mm})$, the system sensitivity drops drastically to less than $20 \%$ of that $(17.2 \mathrm{nF} / \mathrm{kPa})$. Those data suggests that the theoretical model fits the experiments reasonably well under the assumptions. In both cases, the contact area between the droplet and the deformed membrane is smaller than $5 \%$ of the whole chamber area, and therefore can be approximated as a flat interface instead of curved. However, in the smallest devices (with a radius of $1.5 \mathrm{~mm}$ ), the measurements deviate considerably (more than 40\%) from the stimulated values. In this case it is highly possible that the small deformation limit, which is associated with the ratio of the maximum deflection to the radius of the membrane, has been exceeded. In addition, it has been observed that the radius of the curvature of the deflected membrane can no longer be ignored in the smallest sensing unit. Overall, given its 4 th power relation, the radius of the chamber is a determinant factor for the overall mechanical-to-electrical sensitivity.

\section{Influence of Chamber Height}

As predicted by the theoretical analysis, the device sensitivity is inversely proportional to the 2 nd power of the height of the sensing as the membrane deflection dominates, and thus, a lower sensing chamber will lead to a larger contact area at a given droplet volume. Fig. 4 a presents the variations of the sensitivity over four different chamber heights ranging from $140 \mathrm{~m}$ to $350 \mathrm{~m}$, provided the chamber radius of $6.0 \mathrm{~mm}$ and droplet volume of $3 \mathrm{~L}$. As can be seen, the lowest chamber $(140 \mu \mathrm{m}$ in height) shows the highest sensitivity $(374 \mathrm{nF} / \mathrm{kPa})$ due to the presence of the largest contact area at the droplet-electrode interface. As the height of the chamber rises from $140 \mu \mathrm{m}$ to $350 \mu \mathrm{m}$, the device sensitivity decreases from $374 \mathrm{nF} / \mathrm{kPa}$ to $54.5 \mathrm{nF} / \mathrm{kPa}$, closely following the negative quadratic relationship between the sensitivity and the chamber height. Moreover, as the chamber height increases, the separation layer becomes increasingly influential on the overall sensitivity. However, in our cases, the maximal deformation of the separation layer is still one order of magnitude smaller than that of the membrane deflection as expected. In brief, all four sets of the sensitivity measurements are in a good agreement with the theoretical analysis presented above.

\section{Influence of Droplet Volume}

Furthermore, the droplet volume is directly proportional to the system sensitivity. In general, a larger droplet covers more interfacial area with a longer circumferential periphery along the contact, which leads to a higher sensitivity given constant dimensions of a sensing chamber. However, the increased droplet size can also lead to non-linear response as the droplet-electrode interface cannot be approximated as a planar surface. Four different droplet sensing units (of $0.3 \mu \mathrm{L}, 0.6 \mu \mathrm{L}, 1.2 \mu \mathrm{L}$ and $3.6 \mu \mathrm{L}$ ) have been characterized and compared under the identical dimensions of sensing chamber $(6.0 \mathrm{~mm}$ radius and $200 \mu \mathrm{m}$ height $)$, as shown in Fig. $4 \mathrm{~b}$. As the droplet volume increases from $0.3 \mu \mathrm{L}$ to $0.6 \mu \mathrm{L}$, and then to $1.2 \mu \mathrm{L}$, the corresponding sensitivity rises in a linearly proportional manner from $17.2 \mathrm{nF} / \mathrm{kPa}$ to $32.1 \mathrm{nF} / \mathrm{kPa}$, and then to $65.7 \mathrm{nF} / \mathrm{kPa}$. The highest sensitivity $(218 \mathrm{nF} / \mathrm{kPa})$ has been achieved on the sensing droplet of $3.6 \mu \mathrm{L}$ in volume. Again, this matched finding re-confirms the applicability of Eq. 1.

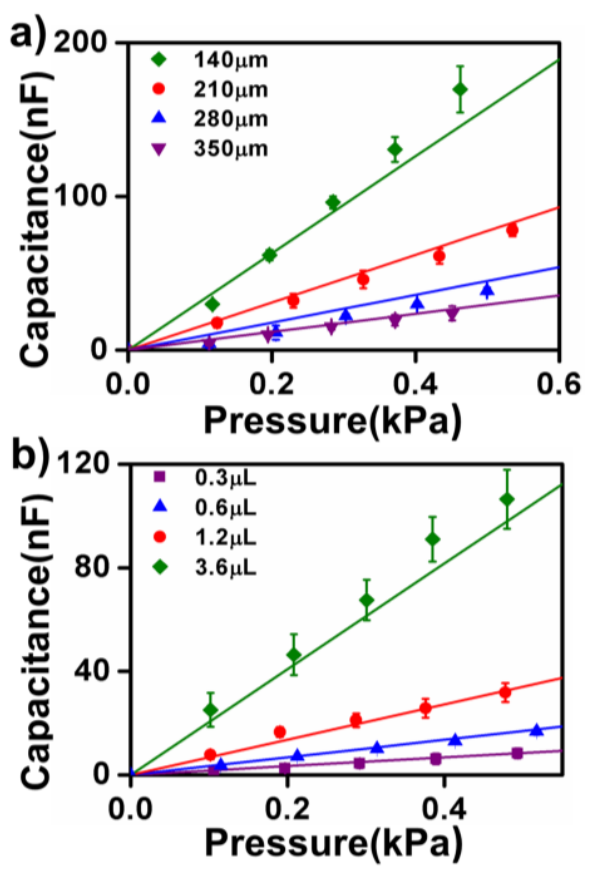

Fig. 4 Influence of (a) heights of the sensing chambers, (b)volume of the sensing droplets on the device sensitivity.

\section{Optimization of Device Sensitivity}

Finally, we have combined the optimal conditions from the above analysis (of $9.0 \mathrm{~mm}$ in radius, $140 \mu \mathrm{m}$ in height and $3 \mu \mathrm{L}$ in droplet volume) and characterized the highest device sensitivity of $1.58 \mathrm{~F} / \mathrm{kPa}$, in comparable to the highest value reported in the literature $(2.24 \mathrm{~F} / \mathrm{kPa}$, using a mercury droplet and an ultrahigh permittivity material), to our best knowledge [2]. Moreover, in the optimal sensing design, the device shows an extremely high pressure resolution of $1.8 \pm 0.4 \mathrm{~Pa}$.

\section{Influences of Temperature and Humidity}

Importantly, the performance of the droplet sensors can be subject to the environmental humidity level. Specifically, the E/G mixture establishes thermodynamic equilibrium with different stable mixing ratios at different humidity levels by either losing (evaporation) or gaining (condensation) aqueous contents to/from the environment [9]. As shown in Fig. 5a, when the humidity level alters, the volume and capacitance of a droplet sensor can substantially deviate from the initial condition (of $25 / 75 \% \mathrm{E} / \mathrm{G}$ ) as the mixture compositions change. However, once a new thermodynamic equilibrium is established, the capacitance will remain stable. Therefore, we can either employ sensing elements with different mixing ratios at different humidity levels or let the sensing element equilibrate with the environment thermodynamically prior to its usage.

In contrast, the environmental temperature fluctuation has only posed minor influence on the interfacial capacitance, in comparison with the resistance-based devices. As shown in Fig. 5b, The electrolyte-electrode interfacial capacitance has been measured at three separate temperatures, $4{ }^{\circ} \mathrm{C}$ (reduced), $25^{\circ} \mathrm{C}$ (regular), and 45 ${ }^{\circ} \mathrm{C}$ (elevated), respectively, from which less than $10 \%$ variation in 
the unit-area capacitance has been observed at the steady state.

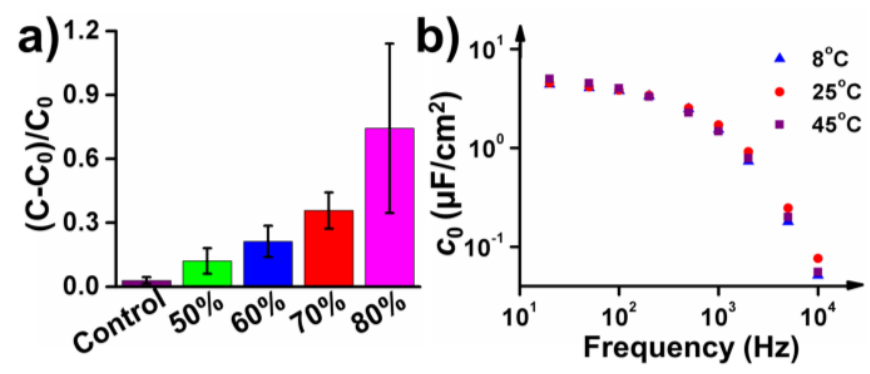

Fig. 5 (a) the humidity influence on the initial capacitance $C_{0}$ and (b) the temperature influence on the unit-area capacitance $c_{0}$.

\section{Demonstration}

To demonstrate the utility of the simply constructed and mechanically flexible droplet sensor with ultrahigh sensitivity and resolution, we applied the device to record blood pressure variations on the skin surface. In this dynamic measurement, a droplet sensor was devised with a built-in chamber size of $6 \mathrm{~mm}$ in radius and $140 \mathrm{~m}$ in height. An electrolyte droplet of $3 \mathrm{~L}$ acted as the sensing element to achieve a facilitated response.

The sensor was attached to the skin surface above the carotid artery (in Fig. 6a) to record the blood pressure wave with a gentle contact force being applied. As shown in Fig. 6b, the minute blood pressure variations were recorded with the maximum value less than $100 \mathrm{~Pa}$ at around $1 \mathrm{~Hz}$. As demonstrated, the ultrahigh sensitivity/resolution, simple configuration and flexible construct of the droplet sensing device could be attractive to a wide range of biomedical applications (e.g., ocular systems, pulmonary, and cardiovascular), where the maximal pressure variations typically range from $2 \mathrm{kPa}$ to $20 \mathrm{kPa}$ and meanwhile, the body conformability and comfortableness are of particular importance for continuous recording [10]-[12].

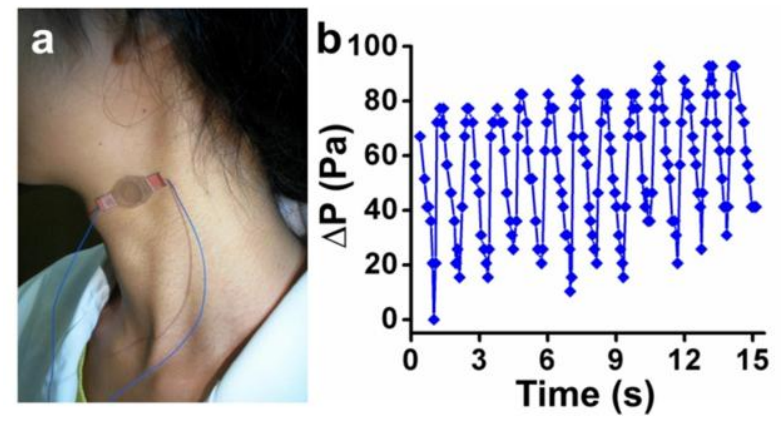

Fig. 6 (a) Applying the capacitive sensor (b) to record the blood pressure variations on the skin surface (above the carotid artery).

\section{CONCLUSIONS}

In this paper, we have presented a novel ultrahigh interfacial capacitive sensor with a very simple architecture and tunable sensitivities. The interfacial sensing principle utilizes the presence of large unit-area EDL capacitance at the elastic droplet-electrode contact. A theoretical model has been proposed to establish the linkage between the geometrical parameters (the radius and height of the sensing chamber and the droplet volume) and the device sensitivity. Taking advantage of ultrahigh sensitivity, simple fabrication, mechanical flexibility and optical transparency, insensitivity to evaporation and thermal noise, the interfacial capacitive sensing devices are expected to be employed in a wide range of biomedical applications where high sensitivity and high flexibility are concurrently required.

\section{ACKNOWLEDGEMENTS}

This work is in part supported the NSF CAREER Award (ECCS-0846502) and EFRI Award (EFRI-0937997) to TP. BN and SX acknowledge the fellowship support from China Scholarship Council (CSC). Authors would also like to acknowledge the samples of ITO-coated membranes generously provided by Southwall Technology. In addition, we would like to thank Mr. Yuzhe Ding for the valuable discussion on the blood pulse measurement.

\section{REFERENCES}

[1] S.C.B. Mannsfeld, B.C.K. Tee, R.M. Stoltenberg, C.V.H.H. Chen, S. Barman, B.V.O. Muir, A.N. Sokolov, C. Reese, and Z. Bao,"Highly Sensitive Flexible Pressure Sensors with Microstructured Rubber Dielectric Layers", Nature Materials, 9, 10 (2010).

[2] E.G. Bakhoum, and M.H.M. Cheng, "Novel Capacitive Pressure Sensor", Journal of Microelectromechanical Systems, 19, 3 (2010).

[3] R.M. Haque, and K.D. Wise, "An Intraocular Pressure Sensor Based on a Glass Reflow Process", Technical Digest of the 2010 Solid-State Sensors, Actuators, and Microsystems Workshop, Hilton Head Isl., SC, 6/6-10/10, Transducer Research Foundation (2010), pp. 49-52.

[4] H. Yuan, H. Shimotani, A. Tsukazaki, A. Ohtomo, M. Kawasaki, and Y. Iwasa, "High-Density Carrier Accumulation in $\mathrm{ZnO}$ Field-Effect Transistors Gated by Electric Double Layers of Ionic Liquids", Advanced Functional Materials, 19, 7 (2009).

[5] R. Lin, P. Taberna, S. Fantini, V. Presser, C.R. Pérez, F. Malbosc, N.L. Rupesinghe, K.B.K. Teo, Y. Gogotsi, and P. Simon, "Capacitive Energy Storage from -50 to $100{ }^{\circ} \mathrm{C}$ Using an Ionic Liquid Electrolyte", The Journal of Physical Chemistry Letters, 2, 19 (2011).

[6] Y. Chuanga, Y. Changa, K. Liua, H. Changb, and T. Yewa, "Electrical Impedimetric Biosensors for Liver Function Detection", Biosensors and Bioelectronics, 28, 1 (2011).

[7] Y. Ding, S. Garland, M. Howland, A. Revzin, and T. Pan, "Universal Nanopatternable Interfacial Bonding", Advanced Materials, 23, 46 (2011).

[8] A.R. Carr, R.E. Townsend, and W.L. Badger, "Vapor Pressures of Glycerol-Water and Glycerol-Water-Sodium Chloride Systems", Industrial and Engineering Chemistry, 17, 6 (1925).

[9] A. K. Ray, R. D. Johnson, A. Souyri, "Dynamic Behavior of Single Glycerol Droplets in Humid Air Streams", Langmuir, 5, 1 (1989).

[10] A.M. Master, L.I. Dublin, and H.H. Marks, "The Normal Blood Pressure Range and Its Clinical Implications", Journal of the American Medical Association, 143, 17 (1950).

[11] J.G. Venegas, R.S. Harris, and B.A. Simon, "A Comprehensive Equation for the Pulmonary Pressure-Volume Curve", Journal of Applied Physiology, 84, 1 (1998).

[12] C.R. Ethier, M. Johnson, J. Ruberti, "Ocular Biomechanics and Biotransport", Annual Review of Biomedical Engineering, 6, 1 (2004).

\section{CONTACT}

*T.Pan, tel: +1-530-754-9508; tingrui@ucdavis.com 